\title{
OLD AND DISPLACED TIBIAL SPINE FRACTURE FIXATION BY OPEN APPROACH-PREFERABLE
}

\section{Orthopaedics}

\section{Dr Dibakar Ray $\quad$ Professor, Iq city medical college,Durgapur}

\section{ABSTRACT}

In the era of athroscopy all intraarticular knee pathology including tibial spine fracture can be managed satisfactory results.In highly displaced situation and in delayed cases open approach is still a gold standard for managing this complex situation to get a saticfactory fracture reduction and fixation and ultimately good functional results.

\section{KEYWORDS}

displaced tibial spine, Open approach,stainless steel wire fixation

\section{Background:}

Adults anterior tibial spine fracture is rare fracture . Undisplaced and minimally displaced (type $1 \& 2$ ) fractures can be treated by closed reduction and long leg cast. To avoid persistent post injury instability and secondary degenerative changes displaced tibial spine fractures (type3) needs surgical intervention mainly arthroscopy guided reuction and fixation. In this study, all highly displaced and delayed(more than 2 weeks) tibial spine fractures (type3) are treated with open reduction and internal fixation with stainless steel wires .

All knee inspected arthroscopic evaluation before open reduction.

\section{INTRODUCTION-}

Literature shows fractures of anterior tibial spine is relatively rare injury in children \& adolescents( 3 per 10000 per year).All displaced anterior tibial spine fracture should be treated by arthroscopy guided reduction and internal fixation followed by proper post operative rehabilitation programme.In some highly displaced and delayed cases open reduction becomes mandatory.

In this series 12 such highly displaced anterior tibial spine fractures were selected for operative treatment with arthroscopic evaluation but open reduction and their results were evaluated.

Follow up shows good result in respect to satisfactory fracture reduction and fixation with healing of all fractures with preservation of normal range of motion of knee

\section{INCLUSION CRITERIA:}

Adult patients presented with fracture more than 2 weeks.

\section{EXCLUSION CRITERIA:}

All children with anterior tibial spine fracture and adults with such fracture less than 2 weeks

\section{MATERIALS \& METHODS:}

1. Total Patients- 12 patient

2. Age -17 to 30 years

3. Time periods- from June 2016 to July 2020

4. Sex ratio- Male female ratio was $4: 1$

5. Mode of injury- Either due to athletic activity or fall from bicycle.

6. Duration since injury -Patient selected was earliest on 16th day and latest 3 month after injury. Of the 12,8 patient were treated 16 day to 30 days after injury and 4 cases were delayed from 1 month to 3 months.

7. Classification system used - Meyers and Mckeever was used to describe the degrees of displacement of tibial spine fractures .

8. Preoperative investigation protocol-

All patients had preoperative knee fracture roentgenogram with MRI scan of knee done to exclude any ligamentous injuries.

All cases who had type 3 fracture were treated by open reduction and internal fixation. None of them were associated with other bony or ligamentous injuries.

9. Preoperative clinical examination-All patients were examined clinically to rule out any associated medial collateral ligament, lateral collateral ligamen, proximal tibia and meniscal injuries.
10.Implant used-Stainless steel wire

11. Operative procedure-

All 12 patients were thoroughly evaluated arthroscopically in repects of intraarticular fracture extention,condition tibial platau,contion of fracture surface,condition of crusiates and menisci and knee corners.After thorough evalution and difficulty faced to reduce the fracture properly by arthroscopic guidance open approach through antero medial arthrotomy was performed .After opening the capsule medially, the medial meniscus was examined. Retraction was used to allow the examination of anterior horn of lateral meniscus. From an anteromedial starting point on the tibial metaphysis, fracture bed were thoroughly freshened from fibrous tissues upto bleeding point drill holes were placed obliquely into the crater in the tibial epiphyses. The fragment was reduced in its bed and wire was passed through the drill holes anteromedially with suture passer keeping the knee in extension and tied securely.

\section{Average operative time-}

$45 \mathrm{mins}$ to $90 \mathrm{~min}$.

13.Post-operative management-

Long leg plaster cast was applied with knee in full extension .The cast was kept for 2 week. Strengthening exercise of the quadriceps and the hamstring were started immediately.Knee rom was started after 2 weeks. Gradual weight bearing was allowed from toe touchdown to full weight bearing from 4 weeks onwards subsequently.

\section{RESULTS:}

The results were evaluated in terms of range of motion, subjective instability and pain. Painless near full range of motion (0 to135 degree)without having subjective instability was marked as excellent( 7 cases).loss of extension (10 degree)without subjective instability and pain was marked as good (4cases)and 20degree or more loss of extension and mild pain during walking(1 case) was marked as fair.All patients were evaluated upto 6 months post surgery until they return to their preoperative daily activities.All patients were able to squat,sit on floor and run satisfactorily.

\section{DISCUSSION:}

All 12 patients we did not find any major associated ligamentous injury .According to Garcia \& Neer et al. 42 fractures of the tibial spine in patients aged 7 to 60 years. Six of them were positive anterior drawer sign indicating associated or cruciate ligament injuries. The basic treatment protocol followed in this study was as recommended by Roberts and Lovell et al. "most anterior tibial spine should be treated by closed reduction \&immobilization in extension and open reduction \&internal fixation for irreducible fractures". No subjective complaints of knee instability was found during follow up, but objective evidence of anterior instability was found in $12 \%$ of cases .As reported by Gronkvist et al. Younger children will compensate somewhat for any anterior instability as the skeleton grows but in older children and adults some anterior instability may persist. Same theory was explained by the study of Willis et,al. who noted that most children have objective evidence of ACL laxity at long term follow up but few have subjective complaints.

In todays practictice arthroscopy assisted fixation technique is the standard treatment for anterior tibial spine fracture. 
Delayed presented and old fracture pose operative challange to properly reduce the fracture as proper bony preparation by arthroscopy assisted technique in many times can not clear all fibrous tissues from fracture surfaces to get proper bleeding bony surface for good reduction and fixation.Even modern bony burr also many times failed to remove all fibrous tissue.Open technique provide adequate prepation of bony surfaces in delayed and old cases at in my hands in adult patients.

Though arthroscopic assisted fixation with suture provide adequate but still inferior to stainless steel wire fixation used in open technique In $90 \%$ adult patients in this study we have achieved excellent to good results as most patients regained an excellent range of motion with loss of extension ranging from 5-15 degree but 1 case $(12 \%)$ did not achieved their terminal 30 degree of extension probably due to improper post operative physiotherapy and rehabilitation, fragment malunion and delayed presentation.

I removed all metal wires after 8 months of operation.

I have not found any superficial or deep infection in this study group.

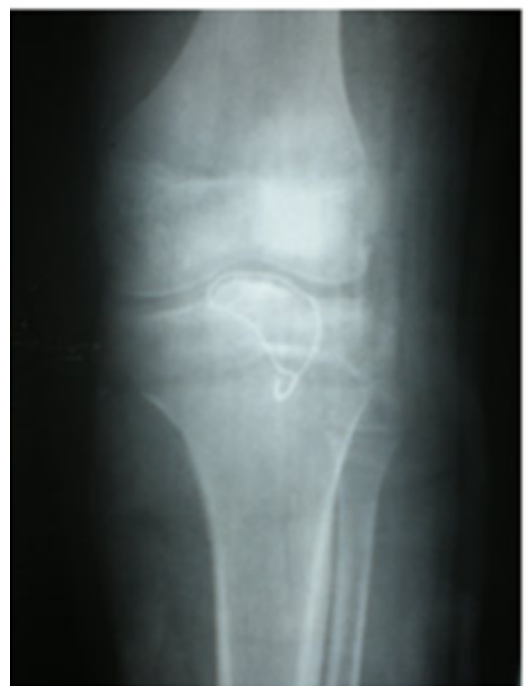

post op ap view

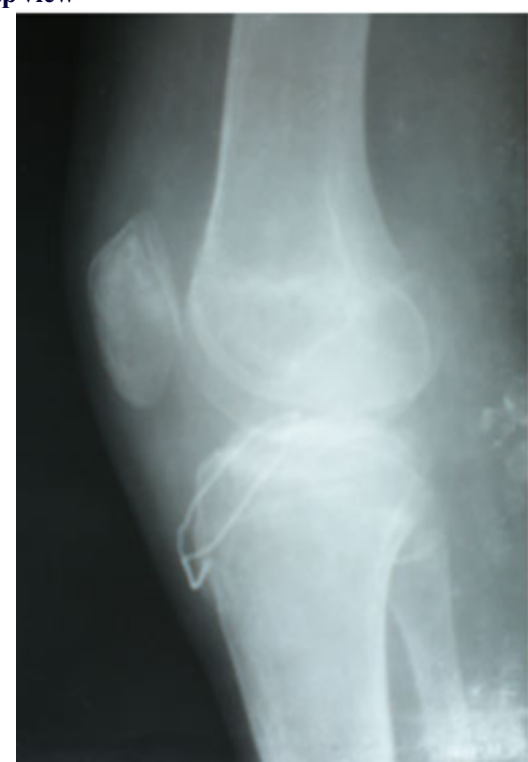

post op lat view

CONCLUSION:

Anterior tibial spine fracture is not a common injury in orthopaedic practice, the diagnosis needs high degree of clinical suspicion and good quality of roentgenogram but other high end investigation to rule out ligament injury. This kind of fracture is more commonly seen in children and adolescent than adults.All Displaced fractures nowadays can be treated by arthroscopic assited fixation technique but delyed and old cases with highly displaced fracture needs arthrotomy and open reduction and internal fixation for proper bony reuction as arhroscopic assisted technique failed to provide proper reduction of fractures. So,in todays arthroscopy era also needs open technique in difficult cases to get good results.

\section{REFERENCES:}

1. Baxter MP,Wiley JJ.Fractures of thetibial spine in children: an evaluation of knee Baxter MP,Wiley JJ.Fractures of thetibial spine
instability.J BoneJoint SurgeryBr 1988;70 228-230

2. Bakalim G, Wilpulla $\mathrm{C}$ : Closed redction of fracture of the tibial spines. INJURY $1974 ; 5: 210-212$

3. Canale ST.Fractures and dislocations. In: Canale ST, Beaty JH, eds Operative Pediatric Orthopedics.

4. Gronkvist H, Hirsch G, Johansson L. fractures of the ant tibial spine in children. J Pediatric Orthop 1984:4:465-468

5. Garcia A, NeerCSII.Isolated fractures of intercondylar eminence of the tibia.Am J Surg 1958; $95: 593-598$

6. Janarv P-M, Westblad P, Johansson C , et al.Long term follow up of ant tibial spine fractures in children J Ped Orthop 1995; 15:63-68.

7. Meyers MH, Mckeever FM Fractures of the intercondylar eminence of yhe tibia.J B J S Meyers MH, Mckeever

8. Pellaci F, Mignani G, Valdiserri L.Fractures of the intercondylar eminence of the tibia in children. Ital JOrthop traumatol 1986; 12: 441-446.

9. Roberts JM, Lovell WW Fractures of the intercondylar eminence of the tibia . JBJS Am $1970 ; 52: 827$

10. Willis RB, Blokker C,Stoll TM, et al .J Ped Orthop 13;361,1993 